\title{
Disparities in Diagnosis and Treatment of Autism in Latino and Non-Latino White Families
}

Katharine E. Zuckerman, MD, MPH, ${ }^{\mathrm{a}, \mathrm{b}}$ Olivia J. Lindly, MPH, ${ }^{\mathrm{a}, \mathrm{c}}$ Nuri M. Reyes, PhD, ${ }^{\mathrm{d}}$ Alison E. Chavez, BA, ${ }^{a}$ Kristy Macias, BA, ${ }^{e, f}$ Kathryn N. Smith, RN, DrPH, ${ }^{e, f}$ Ann Reynolds, MDd, 8

OBJECTIVES: To compare barriers to autism spectrum disorder (ASD) diagnosis and current ASD-related service use among non-Latino white (NLW) families and Latino families with English proficiency (L-EP) or limited English proficiency (L-LEP).

METHODS: We conducted a mixed-mode survey of families of children with confirmed ASD seen at specialty clinics in 3 United States cities. Bivariate and multivariate analyses compared barriers to ASD diagnosis, current service use, and unmet therapy need among NLW, L-EP, and L-LEP families.

RESULTS: Overall, barriers to ASD diagnosis were prevalent: families $(n=352)$ experienced a mean of 8 of 15 barriers to ASD diagnosis. The most prevalent barriers overall were "stress of diagnostic process," "parent knowledge about ASD," and "understanding medical system." Compared with NLW families, L-LEP families were more likely to experience barriers related to knowledge about ASD and trust in providers. Children in L-LEP families also had fewer current therapy hours and more unmet therapy needs than children in NLW families. L-EP families' barriers and treatment services use profile was more similar to NLW than to L-LEP families.

ConcLusions: English proficiency was an important marker for barriers to ASD diagnosis and treatment in Latinos. Increasing ASD-related knowledge and provider trust may decrease disparities in the diagnosis and treatment of ASD among US Latinos.

aDivision of General Pediatrics, Oregon Health and Science University, Doernbecher Children's Hospital, Portland, Oregon; ${ }^{b}$ Oregon Health and Science University-Portland State University School of Public Health, Portland, Oregon; 'College of Public Health and Human Sciences, Oregon State University, Corvallis, Oregon; eDepartment of Pediatrics, University of Southern California, Los Angeles Children's Hospital, Los Angeles, California ${ }^{f}$ University Center for Excellence in Developmental Disabilities, University of Southern California, Los

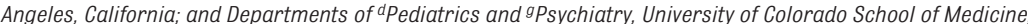
Aurora, Colorado

Dr Zuckerman conceptualized the study, oversaw the project at all stages, and drafted sections of the manuscript; Ms Lindly led the data analysis, assisted in data interpretation, and drafted sections of the manuscript; Dr Reyes and Ms Macias participated in data collection, data analysis, and data interpretation and critically revised and reviewed the manuscript; Ms Chavez organized and analyzed the data, assisted with data interpretation, drafted sections of the manuscript, and prepared the manuscript for publication; Drs Smith and Reynolds led the data collection at 1 site, participated in data analysis and data interpretation, and critically revised and reviewed the manuscript; and all authors approved the final manuscript as submitted and agree to be accountable for all aspects of the work.

DOI: $10.1542 /$ peds.2016-3010

Accepted for publication Feb 9, 2017
WHAT'S KNOWN ON THIS SUBJECT: Racial/ethnic disparities exist in early identification and treatment of autism spectrum disorder (ASD), particularly among Latino children. Although the pervasiveness of these disparities is documented, reasons for these disparities have not been assessed from a family perspective.

WHAT THIS STUDY ADDS: Children in Latino families with parental limited English proficiency experienced different diagnostic barriers, used less ASD treatment, and had more unmet ASD therapy needs than children in English-proficient Latino families or children in non-Latino white families.

To cite: Zuckerman KE, Lindly 0J, Reyes NM, et al. Disparities in Diagnosis and Treatment of Autism in Latino and NonLatino White Families. Pediatrics. 2017;139(5):e20163010 
An estimated 1 in 68 US children has autism spectrum disorder (ASD). ${ }^{1}$ Evidence suggests that prompt ASD diagnosis and treatment improves long-term developmental ${ }^{2,3}$ and family ${ }^{4}$ outcomes. However, multiple studies highlight racial/ethnic disparities in ASD care..$^{5-7}$ Disparities are especially notable among US Latino children: ASD is diagnosed less often among Latino compared with non-Latino white (NLW) children. ${ }^{1}$ Latino children are less often diagnosed before age $4^{8}$ and are diagnosed later than NLW children with similar clinical presentations. ${ }^{9}$ When Latinos are diagnosed, they are more likely to have severe presentations, such as comorbid intellectual disability. ${ }^{5,10}$ After diagnosis, Latinos with ASD receive fewer evidence-based treatments and less medical specialty care than NLWs. ${ }^{6,11}$

Few resources address the reasons for Latino/NLW disparities in ASD care. In Unequal Treatment, ${ }^{12}$ the Institute of Medicine's 2003 report, health care disparities are described as multifactorial in nature: both provider/health care system and patient/family factors play roles. Similar factors may influence ASD disparities: for instance, pediatric providers are less likely to screen Spanish versus English speakers for ASD and feel less comfortable identifying ASD risk when families speak Spanish. ${ }^{13}$ Latinos with Spanish as their primary language receive less family-centered care for ASD than NLW families ${ }^{5,6}$ and are less likely to receive care consistent with families' values. ${ }^{14}$ In addition, commonly used ASD screening ${ }^{15}$ and diagnostic ${ }^{16}$ tools may function differently when used with Latinos, making appropriate screening more difficult.

Less research has examined which patient and family factors contribute to ASD disparities. Maternal education and knowledge about ASD may mediate access to ASD care: families with higher maternal education and ASD knowledge have better access to ASD care regardless of ethnicity. ${ }^{6}$ Latino parents may have less information about ASD generally 17,18 and limited knowledge about diagnostic/ treatment resources ${ }^{6}$ or how to access them. ${ }^{19}$ Community disability stigma and skepticism regarding ASD diagnosis may mediate Latino/NLW disparities. ${ }^{17}$ Child sociodemographic factors, such as public insurance status $^{5}$ or metropolitan residence, ${ }^{20}$ may also impact access to care for children with ASD, although effects on Latinos have not been examined.

To our knowledge, no previous multisite studies have assessed barriers to ASD care among Latino versus NLW families with confirmed ASD. No studies have examined links between barriers experienced and subsequent treatment use. Although English proficiency is a sensitive marker of health care access for Latinos, ${ }^{21}$ no studies have investigated its role in ASD disparities. Thus, we surveyed Latino and NLW families seeking ASD care to determine how barriers to ASD diagnosis differed by ethnicity and parental English proficiency. Among ethnicity/language groups, we sought to identify differences among ASD treatment types and intensity. Finally, we assessed whether barriers to ASD care were associated with disparities in services access among Latinos and NLW families.

\section{METHODS}

\section{Study Sample and Design}

In 2014-2015, we surveyed parents of Latino and NLW children seen at ASD clinics in Los Angeles, California; Denver, Colorado; and Portland, Oregon. Clinics were chosen because they were current or former members of the Autism Speaks Autism Treatment Network and thus had similar ASD diagnostic processes, and because the clinics had high Latino populations. The study enrolled families of children aged 2 to 10 years with an ASD diagnosis confirmed in the previous 5 years. A random sample of eligible medical records was selected at each site and stratified by race/ ethnicity with the use of electronic medical record data, to ensure adequate Latino enrollment. Manual chart audits confirmed that ASD diagnosis included multidisciplinary evaluation and use of Autism Diagnostic Observational Schedule and Diagnostic and Statistical Manual of Mental Disorders, Fourth Edition, Text Revision, or Diagnostic and Statistical Manual of Mental Disorders, Fifth Edition, criteria. ${ }^{22}$ Children in foster care, those with significant comorbid developmental disabilities, those whose families spoke neither English nor Spanish, or who did not identify as Latino or NLW were excluded. Eligible families $(n=489)$ were mailed an English or Spanish survey; families who did not return surveys were offered telephone survey administration by bilingual/bicultural interviewers. Of 489 participants contacted, 370 were surveyed for a composite response rate $^{23}$ of $76.2 \%$. Families who did not indicate race/ethnicity $(n=7)$ on the survey and those with demographic characteristics outside of our eligibility criteria $(n=11)$ were subsequently excluded, yielding an analytic sample of 352 participants. Each institution's institutional review board approved the protocol. Consent was implied by survey completion.

\section{Survey Development}

Previously used and/or validated measures were used when possible. Demographic items were adapted from the 2009/2010 National Survey of Children with Special Health Care Needs ${ }^{24}$ and the US Census American Community Survey ${ }^{25}$ Items on autism services were adapted from the 2011 Survey of Pathways to 


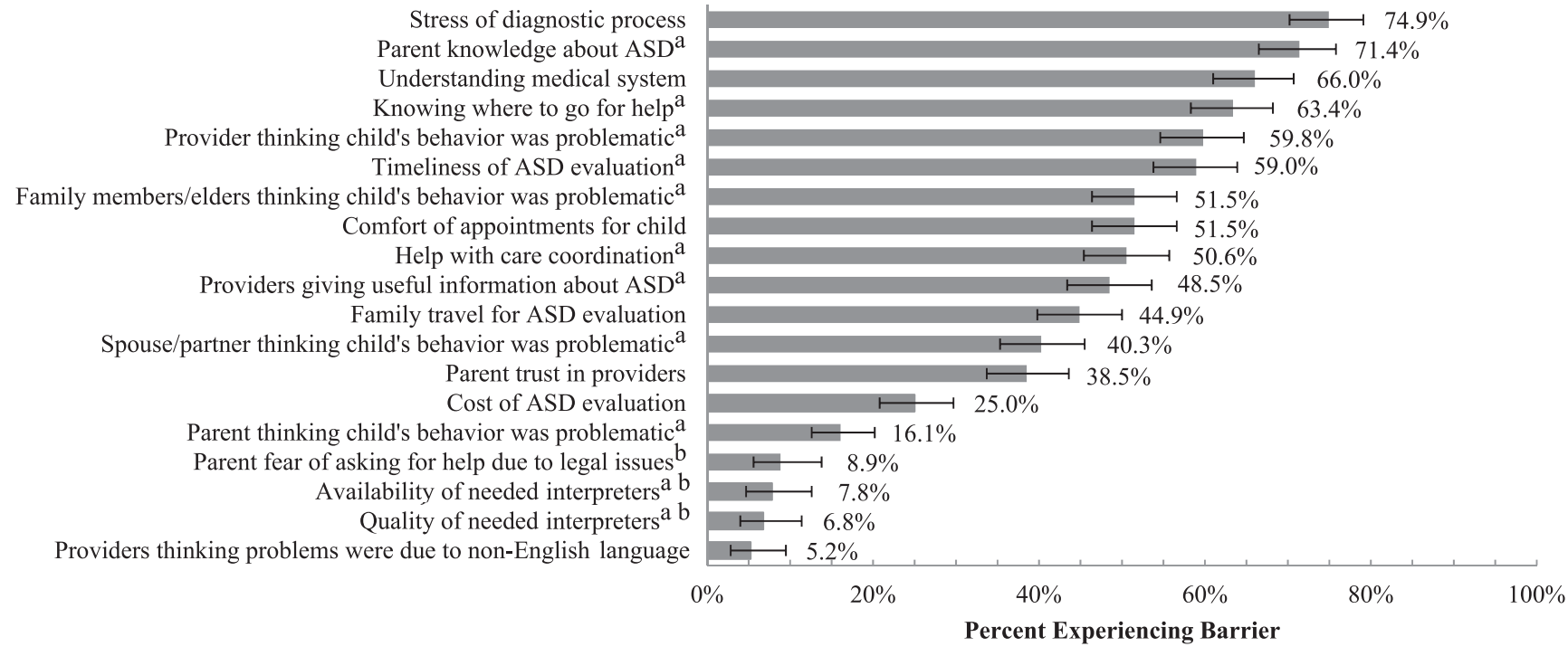

FIGURE 1

Overall prevalence of participants experiencing barriers to ASD care $(n=352) .{ }^{\text {a }}$ Item was reverse coded. ${ }^{\mathrm{b}}$ Item was only assessed among Latinos.

Diagnosis and Services. ${ }^{26}$ Because no validated family-reported measures of barriers to ASD care existed, a new survey measure was developed on the basis of previous qualitative research by our group. ${ }^{17}$ We used English and Spanish cognitive interviews ${ }^{27}$ with parents of children with ASD to iteratively refine item wording, clarify response options, and assess overall survey design. Autism Parent Advisory Committees at 2 sites provided additional feedback on survey wording. The Flesch-Kincaid Grade Level Test was used to adjust items to a sixthgrade reading level. The final survey consisted of 34 items assessing experiences of potential barriers to ASD diagnosis, current therapy use, beliefs about ASD, and demographic information.

\section{Barriers to ASD Diagnosis}

Fifteen items addressed barriers to ASD diagnosis (Fig 1). For each item, participants were asked to "think back about the period before a doctor diagnosed your child with ASD," and were presented with negatively and positively phrased statements (eg, "Before a doctor diagnosed my child with ASD it was difficult to trust doctors' or nurses' advice"). For each statement, participants indicated agreement on a 4-part scale. Four additional items assessed barriers hypothesized as specific to Latinos, such as not speaking English as a primary language and experiencing legal issues. These 4 items included "does not apply" as a response option. A barrier was considered present if a family "somewhat or definitely" agreed with negatively phrased items or "somewhat or definitely disagreed" with positively phrased items; "does not apply" responses were considered equivalent to not experiencing the barrier. A count of the total number of barriers experienced by each family was constructed from the 15 barriers applicable to all families.

\section{Current Treatment Service Use}

Child's current treatment was assessed by using multiple items. A binary item assessed the presence of current Individualized Education Program or Individual Family Service Plan. Parents indicated specific ASD therapy services their child received currently and regularly. Parents then estimated total hours of weekly therapy. Pharmacologic and complementary and alternative medicine treatments for ASD were also assessed. Unmet therapy needs (herein "unmet needs") were assessed by using a 2009/2010 National Survey of Children with Special Health Care Needs item ${ }^{24}$ ("Does your child receive all the therapy service s/he needs?"), which parents rated on a 4-part scale.

\section{Race, Ethnicity, and English Proficiency}

Combined race/ethnicity and English proficiency ("ethnicity/language") was the primary independent variable. It was based on parent report by using items from the US Census American Community Survey. ${ }^{25}$ Three mutually exclusive groups were defined: NLW, Latino English proficient (L-EP), and Latino limited English proficient (L-LEP). Latino ethnicity was determined if the parent reported his/her ethnicity and/or the child's ethnicity was "of Hispanic or Latino origin," regardless of racial category. NLW was determined if the parent reported (1) that his/her race and his/her child's race was white and (2) that both parent and child ethnicity was reported as non-Latino. Parental English proficiency was determined per federal guidelines ${ }^{28}$ by using the item: "How well do you speak English?" Parents responding "very 


\begin{tabular}{|c|c|c|c|c|}
\hline & Overall $(n=352)$ & NLW $(n=163)$ & L-EP $(n=95)$ & L-LEP $(n=94)$ \\
\hline Overall, \% & & 46.3 & 27.0 & 26.7 \\
\hline \multicolumn{5}{|l|}{ Parent/household characteristics } \\
\hline \multicolumn{5}{|l|}{ Respondent's relationship to child, \% } \\
\hline Mother $(n=316)$ & 89.3 & 86.5 & 89.4 & 96.8 \\
\hline Father $(n=27)$ & 8.2 & 9.2 & 9.6 & 3.2 \\
\hline Other $(n=8)$ & 2.5 & 4.3 & 1.1 & 0.0 \\
\hline Number of children per household $(n=346)$, mean \pm SD & $2.1 \pm 1.1$ & $2.0 \pm 1.0$ & $2.2 \pm 1.24$ & $2.4 \pm 1.1$ \\
\hline Number of children with ASD per household $(n=342)$, mean \pm SD & $1.1 \pm 0.4$ & $1.2 \pm 0.5$ & $1.1 \pm 0.3$ & $1.2 \pm 0.6$ \\
\hline \multicolumn{5}{|l|}{ Parent nativity } \\
\hline Always lived in United States $(n=228), \%$ & 64.8 & 90.2 & 82.1 & 3.2 \\
\hline Lived outside the United States ( $n=124), \%$ & 32.3 & 9.8 & 17.9 & 96.8 \\
\hline Years lived in United States, mean \pm SD & $17.2 \pm 8.3$ & $21.9 \pm 13.0$ & $22.4 \pm 9.8$ & $15.5 \pm 6.3$ \\
\hline \multicolumn{5}{|l|}{ Latino parent national origin/heritage, \% } \\
\hline Mexico $(n=110)$ & 78.7 & - & 71.7 & 83.8 \\
\hline Other Latin American countries or Spain $(n=30)$ & 21.3 & - & 28.3 & 16.3 \\
\hline \multicolumn{5}{|l|}{ Family structure, \% } \\
\hline Married or living with partner $(n=278)$ & 79.3 & 80.4 & 77.9 & 78.5 \\
\hline Single $(n=32)$ & 9.0 & 8.6 & 9.5 & 9.7 \\
\hline Other $(n=41)$ & 11.7 & 11.0 & 12.6 & 11.8 \\
\hline Parent education $(n=345)$, mean \pm SD, y & $13.7 \pm 3.9$ & $15.0 \pm 3.6$ & $14.6 \pm 3.0$ & $10.4 \pm 3.4$ \\
\hline Median & 14.0 & 15.0 & 14.0 & 10.5 \\
\hline Range & $0-25$ & $2-25$ & $9-24$ & $0-18$ \\
\hline \multicolumn{5}{|l|}{ Parent employment } \\
\hline Unemployed $(n=178), \%$ & 50.6 & 44.8 & 40.0 & 71.3 \\
\hline Employed $(n=174), \%$ & 49.4 & 55.2 & 60.0 & 28.7 \\
\hline Hours per week, mean \pm SD & $33.9 \pm 12.6$ & $33.4 \pm 12.9$ & $36.5 \pm 12.6$ & $31.0 \pm 10.2$ \\
\hline \multicolumn{5}{|l|}{ Child characteristics } \\
\hline Child's age $(n=343)$, mean \pm SD, y & $5.8 \pm 2.0$ & $5.9 \pm 2.1$ & $5.8 \pm 1.9$ & $5.6 \pm 2.0$ \\
\hline \multicolumn{5}{|l|}{ Child's sex, \% } \\
\hline Male $(n=291)$ & 84.0 & 78.9 & 84.0 & 91.4 \\
\hline Female $(n=57)$ & 16.0 & 21.1 & 16.0 & 8.6 \\
\hline \multicolumn{5}{|l|}{ Child health insurance, \% } \\
\hline Public health insurance only $(n=210)$ & 60.6 & 48.2 & 51.1 & 92.3 \\
\hline Any private health insurance $(n=137)$ & 39.4 & 51.9 & 48.9 & 7.7 \\
\hline \multicolumn{5}{|l|}{ Child ASD severity, \% } \\
\hline Mild $(n=162)$ & 47.1 & 50.3 & 47.9 & 40.7 \\
\hline Moderate or severe $(n=182)$ & 52.8 & 49.7 & 52.1 & 59.3 \\
\hline \multicolumn{5}{|l|}{ Study site characteristics, \% } \\
\hline Site 1 & 31.1 & 53.6 & 22.7 & 23.6 \\
\hline Site 2 & 32.4 & 39.5 & 38.6 & 21.9 \\
\hline Site 3 & 36.5 & 46.1 & 23.3 & 33.6 \\
\hline
\end{tabular}

-, not applicable.

well" were categorized as English proficient. Parents responding "well," "not well," or "not at all" were categorized as limited English proficient.

\section{Covariates}

Child-level covariates included factors known to modify ASD service access, ${ }^{19,29}$ including child age, child sex, parent-reported ASD severity, and child health insurance type. Family-level covariates included the respondent's relationship to the child, parental educational attainment, family structure, parent employment status, and number of children in household.

\section{Statistical Analyses}

Descriptive statistics assessed participant characteristics (Table 1), frequency of barriers to ASD care (Fig 1), and receipt of ASD services (Fig 2). Each site's response characteristics were separately examined before pooling across sites.

Bivariate statistics were computed to compare proportions of L-LEP, L-EP, and NLW families experiencing each barrier and using each treatment.
Adjusted logistic regression was used to determine associations of ethnicity/language with (1) experiencing individual barriers, (2) using each treatment, and (3) experiencing unmet therapy needs (Tables 2 and 3). To determine the expected count of barriers for families of children with ASD according to their ethnicity/language, Poisson regression ${ }^{30}$ was used given the dependent variable type (ie, a count with no negative values) and its distribution (ie, mean $=7.56$ and variance $=7.29$ ). Multinomial logistic regression was used to determine 
ethnicity/language associations with weekly hours of therapy services.

To determine differences between L-LEP and L-EP families compared with NLW families on variables related to treatment use, while examining the effects of barriers experienced, we used multiple approaches. We fit a multinomial regression model to determine associations of weekly therapy hours with ethnicity/language and number of barriers experienced. To determine associations of unmet needs with ethnicity/language and barriers experienced, we fit an adjusted logistic regression model. We tested whether ethnicity/ language modified associations between number of barriers experienced and likelihood of unmet needs.

To minimize type I error while reducing confounding, we used a stepwise backward elimination procedure to include covariates in all multivariable models for elimination $(\alpha=0.15)$ and addition $(\alpha=0.10)$. The covariate selection procedure included all child- and family-level covariates described above. Each multivariable model adjusted for study site to account for site-specific confounding. All analyses were performed in Stata 14.0 (StataCorp, College Station, TX). To account for multiple comparisons, we considered results statistically significant when $P$ values were $<.01 ; P$ values between .01 and .05 were considered of marginal significance.

\section{RESULTS}

\section{Study Sample}

Overall, 352 parents reported data on their family and child with ASD. Per the study design, approximately half of the respondents were NLW (46.3\%). Latino participants were evenly divided between L-EP (27.0\%) and L-LEP (26.7\%). Sites were generally similar in child and

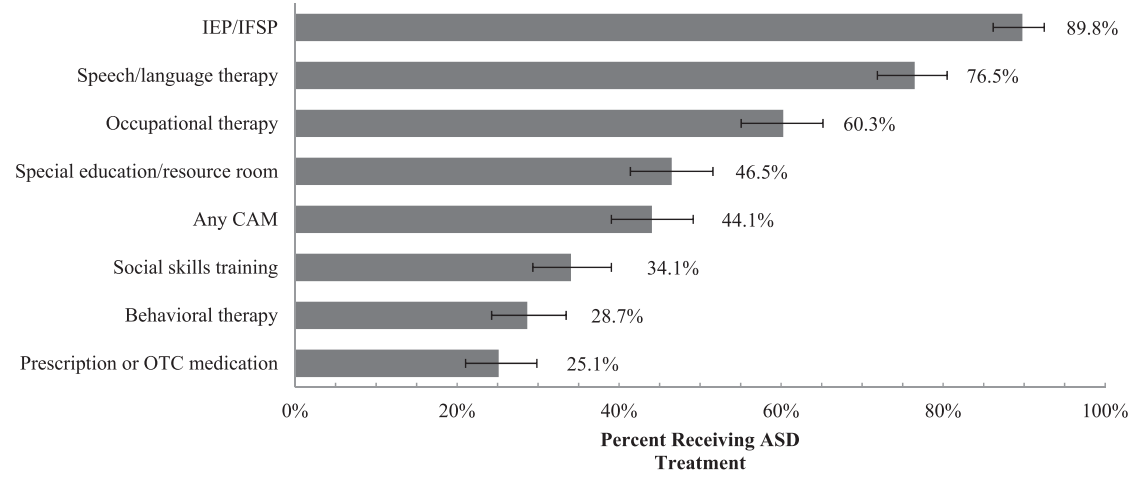

FIGURE 2

Overall prevalence of ASD treatments that participants received $(n=352)$. CAM, complementary and alternative medicine; IEP/IFSP, Individualized Education Program/Individual Family Service Plan; OTC over-the-counter.

family characteristics; site 3 had more Latinos of Mexican origin and more unemployed parents compared with other sites. Compared with other ethnicity/language groups, L-LEP families were more likely to have a mother respondent, have more children per household, have lived outside the United States, have lower parental education, have the respondent be unemployed, and have their child with ASD be publicly insured only (Table 1).

\section{Barriers to ASD Diagnosis}

Families experienced a mean of 8 "somewhat" or "definite" barriers out of 15 barriers to ASD care. Nine barriers were experienced by $>50 \%$ of families. "Stress of diagnostic process" $(74.9 \%)$ was the most frequently reported barrier overall (Fig 1). Among barriers assessed in all families, being L-LEP versus NLW increased the expected number of barriers by $11 \%$, but this association was not statistically significant (adjusted incidence rate ratio: 1.11; 95\% confidence interval: 1.00-1.25; $P=.057$ ). The number of barriers was similar for L-EP and NLW families (adjusted incidence rate ratio: 1.02, 95\% confidence interval: $0.92-1.12$; $P=.76$ ).

The most common barrier among L-LEP and L-EP families was "parent knowledge about ASD" (85.1\% and $72.3 \%$, respectively). The most common barrier among NLW families was "stress of diagnostic process" (78.4\%). "Parent knowledge about ASD" and "parent trust in providers" were significantly more common among L-LEP than among NLW families. "Understanding the medical system" was marginally more common and "cost of ASD evaluation" was marginally less common among L-LEP (versus NLW) families. The only significant difference in specific barriers experienced between L-EP and NLW families was "stress of diagnostic process," which was less common for L-EP families.

None of the 4 barriers hypothesized as being specific to Latinos were as common as the more general 15 barriers. The most common Latinospecific barrier was "parent fear of asking for help due to legal issues." All Latino-specific barriers were more common for L-LEP than for L-EP families, with a large difference in found in "fear of asking for help due to legal issues" (16.0\% vs $2.1 \%$; Table 2).

\section{ASD Treatments, Treatment Dose, and Unmet Treatment Needs}

Overall, $95.7 \%$ of families reported currently using any of the treatments assessed. The most frequently used treatment was speech/language therapy (76.5\%). A total of $28.7 \%$ families reported using behavioral therapy, including applied behavior 


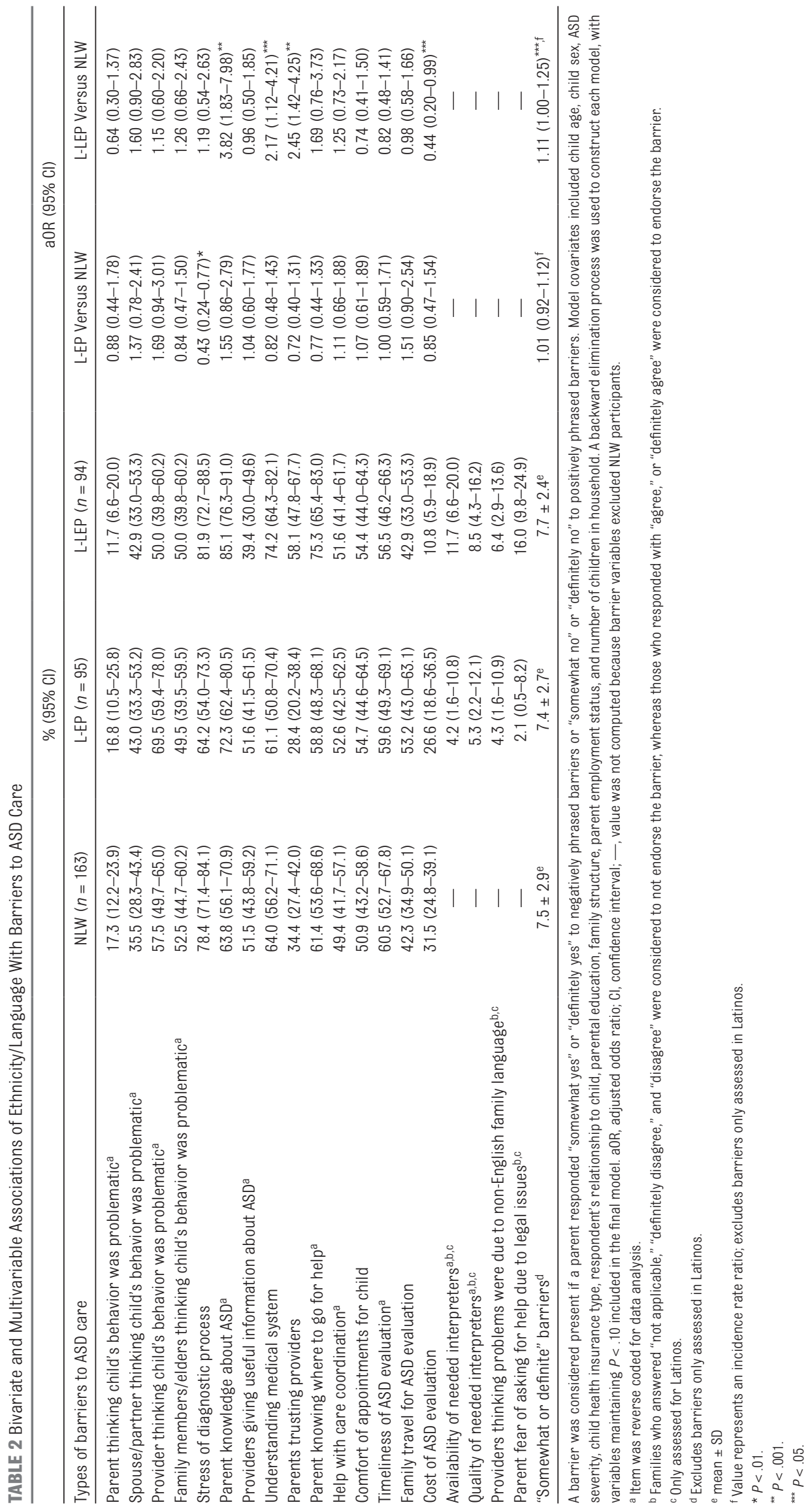


TABLE 3 Bivariate and Multivariable Associations of Ethnicity/Language With Current Services Use

\begin{tabular}{|c|c|c|c|c|c|}
\hline & \multicolumn{3}{|c|}{$\%(95 \% \mathrm{Cl})$} & \multicolumn{2}{|c|}{ Adjusted Ratio $(95 \% \mathrm{Cl})^{\mathrm{a}}$} \\
\hline & $\operatorname{NLW}(n=163)$ & $\mathrm{L-EP}(n=95)$ & L-LEP $(n=94)$ & L-EP versus NLW & L-LEP versus NLW \\
\hline Treatments used for ASD $^{b}$ & & & & \multicolumn{2}{|c|}{ Adjusted OR $(95 \% \mathrm{Cl})^{\mathrm{C}}$} \\
\hline Speech/language therapy & $71.8(64.3-78.2)$ & $81.1(71.8-87.8)$ & 79.8 (70.4-86.8) & $1.48(0.76-2.90)$ & $1.25(0.65-2.39)$ \\
\hline Social skills training & $39.9(32.6-47.6)$ & $27.4(19.3-37.3)$ & $30.9(22.3-41.0)$ & $0.53(0.29-0.97)^{* \star \star}$ & $1.02(0.54-1.93)$ \\
\hline Occupational therapy & $57.7(49.9-65.1)$ & $62.1(51.9-71.3)$ & $60.6(50.4-70.1)$ & $1.11(0.63-1.94)$ & $1.10(0.64-1.87)$ \\
\hline Behavioral therapy ${ }^{d}$ & $33.7(26.9-41.4)$ & $33.7(24.9-43.8)$ & $14.9(9.0-23.7)$ & $0.87(0.47-1.61)$ & $0.63(0.27-1.50)$ \\
\hline $\begin{array}{l}\text { Special education/resource } \\
\text { room }\end{array}$ & $50.3(42.6-58.0)$ & $42.1(32.5-52.3)$ & $46.8(36.9-57.0)$ & $0.60(0.34-1.04)$ & $0.72(0.39-1.35)$ \\
\hline Any CAM & $50.3(42.6-58.0)$ & $42.1(32.5-52.3)$ & $38.30(29.0-48.6)$ & $0.81(0.46-1.40)$ & $0.57(0.33-0.99)^{\star \star \star}$ \\
\hline Prescription or OTC meds & $25.8(19.6-33.1)$ & $29.5(21.1-39.5)$ & $20.2(13.2-29.6)$ & $1.15(0.59-2.25)$ & $0.81(0.39-1.69)$ \\
\hline IEP/IFSP & $88.2(82.2-92.4)$ & $94.7(87.9-97.8)$ & $88.9(80.5-94.0)$ & $0.46(0.16-1.30)$ & $2.21(0.72-6.80)$ \\
\hline Unmet need for therapy & $40.9(33.5-48.7)$ & $51.6(41.5-61.5)$ & $60.4(50.0-70.0)$ & $1.55(0.89-2.69)$ & $2.12(1.21-3.70)^{*}$ \\
\hline \multicolumn{4}{|l|}{ Therapy dose } & \multicolumn{2}{|c|}{ Adjusted RR Ratio $(95 \% \mathrm{CI})^{\mathrm{e}}$} \\
\hline$<1 \mathrm{~h}$ or no weekly therapy & $12.4(8.1-18.5)$ & $13.7(8.1-22.2)$ & $26.1(18.0-36.4)$ & $1.61(0.61-4.25)$ & $4.51(1.73-11.77)^{*}$ \\
\hline $1-10 \mathrm{~h}$ weekly therapy & $64.0(56.2-71.1)$ & $63.2(53.0-72.3)$ & $59.1(48.5-68.9)$ & $1.39(0.71-2.72)$ & $1.73(0.80-3.73)$ \\
\hline$\geq 11 \mathrm{~h}$ weekly therapy & $23.6(17.6-30.8)$ & $23.2(15.7-32.8)$ & $14.8(8.7-23.9)$ & 1.00 & 1.00 \\
\hline
\end{tabular}

CAM, complementary and alternative medicine; Cl, confidence interval; IEP/IFSP, Individual Education Program/Individual Family Service Plan; OR, odds ratio; OTC, over-the-counter; RR, relative risk.

a Model covariates included child age, child sex, ASD severity, child health insurance type, respondent's relationship to child, parental education, family structure, parent employment status, and number of children in household. A backward elimination process was used to construct each model, with variables maintaining $P<.10$ included in the final model.

${ }^{b}$ Child was considered to be receiving therapy if the therapy was delivered currently on a regular basis.

${ }^{\mathrm{C}}$ The values in this column represent adjusted ORs for all ASD treatments, with the referrent being if the child did not receive the respective treatment.

¿Behavioral therapy includes applied behavioral analysis and "other behavioral therapies."

e The values in this column represent adjusted RR ratios estimated from a multinomial logistic regression model in which $\geq 11$ therapy hours per week was the referent category.

${ }^{\star} P<.01$.

${ }^{* * *} P<.05$.

TABLE 4 Associations of Barriers to ASD Diagnosis and Treatment With Services Use

\begin{tabular}{|c|c|c|c|}
\hline & \multicolumn{2}{|c|}{ Multinomial Logistic Model $(n=328)$} & \multirow{2}{*}{$\begin{array}{l}\text { Logistic Model } \\
\qquad(n=337)\end{array}$} \\
\hline & $\begin{array}{l}1-10 \text { Hours of Therapy per } \\
\text { Week Versus } \geq 11 \text { Hours of } \\
\text { Therapy, Adjusted RR Ratio } \\
(95 \% \mathrm{Cl})\end{array}$ & $\begin{array}{l}<1 \text { or No Hours of Therapy } \\
\text { per Week Versus } \geq 11 \text { Hours } \\
\text { of Therapy, Adjusted RR Ratio } \\
\qquad(95 \% \mathrm{Cl})\end{array}$ & \\
\hline $\begin{array}{c}\text { Number of barriers } \\
\text { experienced }\end{array}$ & $0.95(0.86-1.05)$ & $0.95(0.83-1.09)$ & $1.22(1.11-1.33)$ \\
\hline$P$ & .32 & .48 & $<.001$ \\
\hline \multicolumn{4}{|l|}{$\begin{array}{c}\text { Parent Latino } \\
\text { ethnicity }\end{array}$} \\
\hline NLW & 1.00 & 1.00 & 1.00 \\
\hline L-EP & $1.44(0.74-2.80)$ & $1.55(0.60-4.02)$ & $1.50(0.87-2.59)$ \\
\hline$P$ & .29 & .36 & .15 \\
\hline L-LEP & $1.82(0.84-3.94)$ & 4.59 (1.76-11.97) & $1.95(1.12-3.39)$ \\
\hline$P$ & .13 & .002 & .02 \\
\hline
\end{tabular}

All models included number of barriers, parent ethnicity, and site. A stepwise backward elimination procedure was used to select the remaining child and/or family covariates for each multivariable model. $\mathrm{Cl}$, confidence interval; $0 \mathrm{R}$, odds ratio; RR, relative risk.

analysis; $89.8 \%$ of families reported their child had an Individualized Education Program/Individual Family Service Plan (Fig 2). There were few differences in type of therapy by ethnicity/language; however, L-LEP families were less likely to use complementary and alternative medicine and L-EP families were less likely to use social skills training (versus NLW families) (Table 3).

Most children received 1 to 10 hours of weekly therapy for ASD. Compared with NLW children, L-LEP children were more likely to have $<1$ or no hours of weekly therapy versus $\geq 11$ weekly therapy hours. There were no significant differences in therapy dose between L-EP and NLW children. Unmet need for therapy was experienced by the majority of families, but was significantly more likely among L-LEP versus NLW families (Table 3).

\section{Associations of Ethnicity/Language With Barriers, Therapy Dose, and Unmet Needs}

When the number of barriers to ASD care was held constant in multivariable models, L-LEP children were still less likely to have any treatment hours for ASD $(<1$ or no hours) compared with NLW children. Similarly, L-LEP families were more likely than NLW families to have unmet needs after adjustment for number of barriers (Table 4). This relationship is shown in Supplemental Fig 3: as the number of barriers to ASD care increases, Latinos (L-EP and L-LEP) were increasingly more likely than NLW families to have unmet needs. 


\section{DISCUSSION}

This study assessed racial/ethnic and language differences in barriers to care and service use in a multisite sample of children with confirmed ASD. The study found that although all families experienced significant barriers to ASD care, L-LEP families experienced some barriers disproportionately, had the highest levels of unmet therapy needs, and the lowest weekly therapy hours. Higher numbers of barriers to care were associated with more unmet needs, particularly among Latinos. Study findings indicate the L-LEP families especially struggle in accessing ASD diagnostic and treatment resources.

The most frequent barrier for L-LEP families, and the barrier differing most from NLW families, was "parent knowledge about ASD," which was experienced by $>85 \%$ of L-LEPs. The finding confirms qualitative work revealing a low awareness of ASD and its symptoms in Spanishspeaking Latino communities, ${ }^{17,18}$ even as overall ASD awareness has become widespread. Similarly, "parent knowing where to go for help" was slightly more common among L-LEP compared with NLW families, suggesting the information about the ASD diagnostic process is important for L-LEP families. The study found significant differences in therapy hours and unmet needs by ethnicity/language, suggesting access to all types of therapy services remains a significant challenge for L-LEP families.

It is notable that even after adjustment for number of barriers, L-LEP children were still less likely to receive therapy. In addition, as the number of barriers increased, unmet needs affected Latinos disproportionately. Findings suggest that although more barriers are associated with unmet therapy needs for Latinos, they do not fully explain disparities in service use. Some barriers studied may be more important than others as mediators of access to care.

Findings are also notable for barriers that did not differ between NLW families and Latinos. No ethnicity/ language differences were found in rates of caregivers or providers who thought a child's early behavior was problematic, even though this is sometimes cited as a reason Latinos delay seeking ASD care. ${ }^{31,32}$ Likewise, logistical (eg, travel) and economic (eg, cost) barriers were less common than knowledge gaps about ASD and the diagnostic process, and factors such as interpreting services and legal concerns played relatively minor roles. Although these factors are often cited as deterrents to ASD care in minorities, our findings suggest that factors such as ASD knowledge and health care system navigability may be more important intervention targets. More broadly, 9 of 15 barriers studied were experienced by at least $50 \%$ of the sample, and mean number of barriers experienced was nearly 8. Thus, whereas aspects of ASD health care access were challenging for L-LEP families, many challenges were shared by most families of children with ASD. Because several of the more disparate barriers were prevalent in all groups, targeting these barriers might both improve overall care quality and reduce disparities.

Findings suggest opportunities for remediation of disparities in access to ASD services. Because "parent knowledge about ASD" and "understanding the medical system" showed significant racial/ ethnic differences, campaigns and community awareness activities in Latino communities might increase knowledge about ASD and reduce disparities. To be effective, campaigns should use simple, clear messaging about ASD and steps parents should take to access care. Given "trust in providers" was also more problematic for L-LEP compared with NLW families, messages should be delivered in culturally competent ways or via trusted brokers (eg, community members, Latino parents of children with ASD). Family navigation, which has shown promise for ethnically diverse families of children with ASD, ${ }^{33}$ or a Spanish-language ASD-specific helpline may assist L-LEP families better access services. Although use of the Internet is lower in families with limited English proficiency, ${ }^{34}$ high-quality, low-reading-level Spanish-language Web resources may also help L-LEP families. Diagnostic and therapy providers, particularly those in areas with large Latino populations, should employ bilingual, bicultural staff to better engender trust and assist with connections to ASD services. Finally, education of pediatric providers and community workers in identifying early signs of ASD, how to communicate about ASD in culturally competent ways, and how to most effectively connect families with services might improve care for Latinos.

The study had limitations. Barriers other than those studied may be important in ASD service use. Responses were ascertained by retrospective parent report. Recall bias may affect reporting, although it may not differ by ethnicity/ language. We minimized recall bias by surveying families of children diagnosed during the prior 5 years. The study assessed experiences of families whose children ultimately obtained an ASD diagnosis and who were diagnosed at academic medical centers. Consequently, the findings may underestimate barriers to care. Finally, most Latino participants were of Mexican origin. Previous research suggests that Latino health outcomes vary by national origin ${ }^{35}$; however, we lacked adequate numbers of subjects of other origins to conduct subanalyses. 


\section{CONCLUSIONS}

To our knowledge, this is the largest and only multisite study assessing Latino/white disparities in diagnosis and treatment among children with confirmed ASD. Results suggest that health care quality among Latino families of children with ASD remains problematic. The study brings better understanding of specific problems in the diagnosis and treatment process, which may ultimately improve health care quality and promote health equity among children with ASD.

\section{ACKNOWLEDGMENTS}

We thank Christina Nicolaidis and Thomas Becker for their assistance with overall study design, Angie Mejia for her assistance with survey development, and Ann Folan, Lori Ball and Maritza Cobian for their assistance with data collection.

\section{ABBREVIATIONS}

ASD: autism spectrum disorder

L-EP: Latino English proficiency/ proficient

L-LEP: Latino limited English proficiency/proficient

NLW: non-Latino white

Address correspondence to Katharine E. Zuckerman, MD, MPH, Division of General Pediatrics, Oregon Health and Science University, 707 SW Gaines St, Mail Code CDRC-P, Portland, OR 97239. E-mail: zuckerma@ohsu.edu

PEDIATRICS (ISSN Numbers: Print, 0031-4005; Online, 1098-4275).

Copyright (C) 2017 by the American Academy of Pediatrics

FINANCIAL DISCLOSURE: The authors have indicated they have no financial relationships relevant to this article to disclose.

FUNDING: This project was funded by grant K23MH095828 from the National Institute of Mental Health (principal investigator: Dr Zuckerman). Funded by the National Institutes of Health $(\mathrm{NIH})$.

POTENTIAL CONFLICT OF INTEREST: The institutions of Drs Reynolds, Smith, and Zuckerman and Ms Chavez have each received research funding from Autism Speaks; the other authors have indicated they have no potential conflicts of interest to disclose.

\section{REFERENCES}

1. Christensen DL, Baio J, Braun KV, et al Prevalence and characteristics of autism spectrum disorder among children aged 8 years — autism and developmental disabilities monitoring network, 11 sites, United States, 2012. MMWR Surveill Summ. 2016;65(3):1-23

2. Reichow B, Barton EE, Boyd BA, Hume K Early intensive behavioral intervention (EIBI) for young children with autism spectrum disorders (ASD). Cochrane Database Syst Rev. 2012;10:CD009260

3. Rogers SJ, Vismara LA. Evidence-based comprehensive treatments for early autism. J Clin Child Adolesc Psychol. 2008;37(1):8-38

4. Schieve LA, Blumberg SJ, Rice C, Visser SN, Boyle C. The relationship between autism and parenting stress. Pediatrics 2007;119(suppl 1):S114-S121

5. Liptak GS, Benzoni LB, Mruzek DW, et al. Disparities in diagnosis and access to health services for children with autism: data from the National Survey of Children's Health. J Dev Behav Pediatr. 2008;29(3):152-160

6. Magaña S, Lopez K, Aguinaga A, Morton $\mathrm{H}$. Access to diagnosis and treatment services among Latino children with autism spectrum disorders. Intellect Dev Disabil. 2013;51(3):141-153

7. Parish S, Magaña S, Rose R, Timberlake M, Swaine JG. Health care of Latino children with autism and other developmental disabilities: quality of provider interaction mediates utilization. Am J Intellect Dev Disabil. 2012;117(4):304-315

8. Christensen DL, Bilder DA, Zahorodny $W$, et al. Prevalence and characteristics of autism spectrum disorder among 4-year-old children in the autism and developmental disabilities monitoring network. J Dev Behav Pediatr. 2016;37(1):1-8

9. Mandell DS, Wiggins LD, Carpenter LA, et al. Racial/ethnic disparities in the identification of children with autism spectrum disorders. Am J Public Health. 2009;99(3):493-498

10. Developmental Disabilities Monitoring Network Surveillance Year 2010 Principal Investigators; Centers for Disease Control and Prevention. Prevalence of autism spectrum disorder among children aged 8 years - autism and developmental disabilities monitoring network, 11 sites, United States, 2010. MMWR Surveill Summ. 2014;63(2):1-21

11. Broder-Fingert $S$, Shui A, Pulcini CD, Kurowski D, Perrin JM. Racial and ethnic differences in subspecialty service use by children with autism Pediatrics. 2013;132(1):94-100

12. Committee on Understanding and Eliminating Racial and Ethnic Disparities in Health Care; Board on Health Sciences Policy; Institute on Medicine. Unequal Treatment: Confronting Racial and Ethnic Disparities in Healthcare. Washington, DC: National Academies Press; 2003

13. Zuckerman KE, Mattox K, Donelan K, Batbayar 0, Baghaee A, Bethell C. Pediatrician identification of Latino children at risk for autism spectrum disorder. Pediatrics. 2013;132(3):445-453

14. Magaña S, Parish SL, Son E. Have racial and ethnic disparities in the quality of health care relationships changed for children with developmental disabilities and ASD? Am J Intellect Dev Disabil. 2015;120(6):504-513 
15. Kimple KS, Bartelt EA, Wysocki KL, Steiner MJ. Performance of the modified checklist for autism in toddlers in Spanish-speaking patients. Clin Pediatr (Phila). 2014;53(7):632-638

16. Vanegas SB, Magaña S, Morales M, McNamara E. Clinical validity of the ADI-R in a US-based Latino population. J Autism Dev Disord. 2016;46(5):1623-1635

17. Zuckerman KE, Sinche B, Mejia A, Cobian M, Becker T, Nicolaidis C. Latino parents' perspectives on barriers to autism diagnosis. Acad Pediatr. 2014;14(3):301-308

18. Zuckerman KE, Lindly OJ, Bethell $\mathrm{CD}$, Kuhlthau K. Family impacts among children with autism spectrum disorder: the role of health care quality. Acad Pediatr. 2014;14(4):398-407

19. Thomas KC, Ellis AR, McLaurin C, Daniels J, Morrissey JP. Access to care for autism-related services. J Autism Dev Disord. 2007;37(10):1902-1912

20. Kalkbrenner AE, Daniels JL, Emch M, Morrissey J, Poole C, Chen JC. Geographic access to health services and diagnosis with an autism spectrum disorder. Ann Epidemiol. 2011;21(4):304-310

21. Yu SM, Singh GK. Household language use and health care access, unmet need, and family impact among CSHCN. Pediatrics. 2009;124(suppl 4):S414-S419
22. American Psychiatric Association. Diagnostic and Statistical Manual of Mental Disorders(DSM-5). Arlington, VA: American Psychiatric Publishing; 2013

23. American Association for Public Opinion Research. Response rate-an overview. Available at: www.aapor.org/ Education-Resources/For-Researchers/ Poll-Survey-FAQ/Response-Rates-AnOverview.aspx. Accessed July 27, 2016

24. National Center for Health Statistics. Frequently asked questions: 2009-2010 national survey of CSHCN. Available at: https://www.cdc.gov/nchs/data/ slaits/nscshcnfaqs2009.pdf. Accessed December 13, 2016

25. Bureau of US Department of Commerce. U.S. census American communities survey. 2015. Available at: https://www.census.gov/programssurveys/acs/. Accessed June 28, 2016

26. National Center for Health Statistics. Frequently asked questions: 2011 survey of pathways to diagnosis and services. Available at: www.cdc.gov/ nchs/data/slaits/PathwaysFAQ.pdf. Accessed December 13, 2016

27. Fowler FJ. Improving Survey Questions Thousand Oaks, CA: Sage Publications; 1995

28. US Federal Government. Limited English proficiency: frequently asked questions. Available at: https:// www.lep.gov/faqs/faqs.html\#0neQ1. Accessed June 28, 2016
29. Zablotsky B, Pringle BA, Colpe LJ, Kogan MD, Rice C, Blumberg SJ. Service and treatment use among children diagnosed with autism spectrum disorders. J Dev Behav Pediatr. 2015;36(2):98-105

30. Long JS, Freese J. Regression Models for Categorical Dependent Variables Using Stata. 2nd ed. College Station, TX: Stata Press; 2006

31. Blanche El, Diaz J, Barretto T, Cermak SA. Caregiving experiences of Latino families with children with autism spectrum disorder. Am J Occup Ther. 2015;69(5):6905185010p1-69051850 $10 \mathrm{p} 11$

32. Mandell DS, Novak M. The role of culture in families' treatment decisions for children with autism spectrum disorders. Ment Retard Dev Disabil Res Rev. 2005;11(2):110-115

33. Feinberg E, Abufhele M, Sandler J, et al. Reducing disparities in timely autism diagnosis through family navigation: results from a randomized pilot trial. Psychiatr Serv. 2016;67 (8):912-915

34. Knapp C, Madden V, Wang H, Sloyer $P$, Shenkman E. Internet use and eHealth literacy of low-income parents whose children have special health care needs. J Med Internet Res. 2011;13(3):e75

35. Zsembik BA, Fennell D. Ethnic variation in health and the determinants of health among Latinos. Soc Sci Med. 2005;61(1):53-63 